\title{
Robust phase demodulation of interferograms with open or closed fringes
}

(Submitted to JOSA A, 2004)

\author{
Mariano Rivera \\ Centro de Investigacion en Matematicas A.C., \\ Apdo. Postal 402, Guanajuato, Gto. Mexico 36000 \\ mrivera@cimat.mx
}

\begin{abstract}
Analysis of fringe patterns with partial-field and/or closed fringes is still a challenging problem that requires the development of robust methods. This paper presents a method for fringe pattern analysis with those characteristics. The method is initially introduced as a phase refinement process for computed coarse phases, as those obtained from partial-field patterns with a full-field method for open fringes analysis. Based on the phase refinement method, it is proposed a propagative scheme for phase retrieval from closed-fringe interferograms. The algorithm performance is evaluated in real data with no homogeneous illumination components and shows a superior performance than state of the art methods.
\end{abstract}

\section{Introduction}

In interferogram analysis, we often face the problem of computing phase from interferograms with partial-field fringes, i.e. inteferograms where the fringe pattern is visible in a subregion of the image. The interferogram analysis with full-field methods ${ }^{1,2}$ introduces artifacts at the border of the interferogram region. One common solution to this problem is to use phase stepping techniques. ${ }^{3}$ However, such methods are not applicable in fast transients phenomena. Other solution is based on inverse problem theory. In his framework, the robust quadrature filter (RQF) method 4 proposed a regularized solution that, effectively, recovers the wrapped phase in irregular domains, with an additional computational cost required for minimizing the cost function. Other challenging problem in the analysis of fringe patterns is the processing of closed fringe patterns. This problem has captured the attention of researchers in fringe analysis for the last years, see Refs. [5]-[14]. Although there has been important advances, the performance of the reported methods is seriously reduced if the processed fringe pattern has a wide band, it is not normalized and it is corrupted by noise.

In this paper, we present two algorithm for dealing with the problems of: 
1. Phase refinement. Our method uses the fringe pattern as data and a phase computed with an open-fringes full-field analysis method (as ones in Refs. [1,2]) as initial guess. The artifacts, in initial phase, are systematically reduced by an iterative algorithm.

2. Phase recovering from closed-fringe interferograms. This new method for closed-fringes analysis is the most important contribution of the paper and it is based on the previously proposed refinement method (last point). As previous reported methods, our method uses a propagative scheme. The knowledge propagation scheme from a seed site to the whole interferogram was reported in ${ }^{5}$ for the first time: they propagated the sign of absolutes frequency. Since then, this is the underlying idea in closed fringe analysis methods: propagation of frequency signs or entire phase. ${ }^{6,8,9,12,14}$ In our method, a quality map leads the propagation path. The needed of a quality map was noted by Villa et al. ${ }^{8}$ Afterward, Servin et al. used a binarization of the fringe pattern as quality map in the fringe follower method. ${ }^{9}$ Unfortunately, the performance of the fringe quantization is degraded by noise and spatial variations in background/contrast illumination components. Differently to the last mentioned techniques, our quality map does not constraint the phase propagation to follow fringes and at the same time it avoids saddle and stationary points - that is well known may induce propagative algorithm to carry out a wrong phase. Finally, it is important to remark that our method is based on alternated minimizations of quadratic cost functions, i.e. in alternated solution of linear systems. As result, we have a numerically stable algorithm.

Now, we introduce our notation. An image is a regular lattice of pixels, the set of such pixels is denoted by $L$ and the pixel position is denoted by $r=[x, y]^{T}$. Then a fringe pattern, $g$, with or without closed fringes, is modeled by

$$
g_{r}=a_{r}+b_{r} \cos \left(f_{r}\right)+\eta_{r},
$$

for all $r \in T \subseteq L$; where $T$ is the subregion that contains the observed fringe pattern, $a$ is the background illumination component, $b$ is the fringe contrast, $f$ is the unknown phase and $\eta$ represents additive independent noise. In general, the terms $a$ and $b$ are also unknown and need be estimated. If $a$ and $b$ have limited bandwidth, i.e. they have smooth spatial variations, then a preprocessing of the fringe pattern can reduce significantly the contribution of $a, b$ and $\eta$ in (1). ${ }^{9,14}$ If such preprocess is successfully achieved, then one obtains a normalized fringe pattern:

$$
\hat{g}_{r}=\hat{b}_{r} \cos f_{r},
$$

where $\hat{b}$ is an estimation of $b$. Afterward, on can use an existing method for demodulating closed-fringe patterns of the form $\hat{g}$, for instance the regularized phase-tracking method (RPT).${ }^{9,14}$ More recently, Legarda et al. ${ }^{12}$ modified the RPT method for computing $b$ and $f$, 
simultaneously. Although our formulation admits the straightforward generalization for the joint estimation of $b$ and $f$, in this paper, we focus in the estimation of the phase. We prefer the form (2), instead of the form $\hat{g}_{r}=\cos f_{r}$, because it avoids the component-wise division by small values $\hat{b}_{r}$ at those noisy and low contrast sites.

The paper is organized in two part: sections 2 and 3, respectively. In section 2 introduces the method for refining a initial phase. Section 3 extends the phase refinement methods for computing phases from closed fringes. Numerical experiments that demonstrate the methods performance are presented in subsections of the respective parts. Finally, our conclusions are given in section 4 .

\section{Robust Phase Refinement Method}

\section{A. Mathematical Formulation}

The phase refinement method assumes that there are available approximations of contrast, $\hat{b}$, and phase, $\psi$, in the domain of interest, $R$ (we assume in this point that $R=T$, but in general $R \subseteq T$ ). Such approximations can be computed using standard fringe analysis methods, for instance, the Discrete Fourier Transform based method ${ }^{1}$ or the one reported in Ref. ${ }^{2}$ Such methods introduce artifacts at the image borders or along phase discontinuities. So that, in order to compute the true phase, $f=\psi+\phi$, we need to estimate a residual phase, $\phi$. Now, we suppose that $\psi$ is close enough to $f$ such that the first order Taylor series approximate very well the model, i.e.

$$
E\left(\phi_{r} ; \psi_{r}\right) \stackrel{\text { def }}{=} \hat{g}_{r}-\hat{b}_{r}\left(\cos \psi_{r}-\phi_{r} \sin \psi_{r}\right) \approx 0
$$

As it is common to have large residuals at the edges, or at noisy regions, we relax the constraint (3) to be small almost everywhere: Then those pixels with large residuals need be treated as outliers. Therefore, we propose to compute the residual phase, $\phi$, and an outliers detector field, $\omega$, as the minimizers of the regularized (robust) half-quadratic cost function (see Refs. $\left.{ }^{15}-20\right)$ :

$$
\begin{aligned}
U_{1}(\phi, \omega ; \psi)= & \sum_{r \in R}\left[\omega_{r}^{2} E^{2}\left(\phi_{r} ; \psi_{r}\right)+\mu\left(1-\omega_{r}\right)^{2}\right] \\
& +\lambda \sum_{\langle q, r, s\rangle \in R}\left[\psi_{q}+\phi_{q}-2\left(\psi_{r}+\phi_{r}\right)+\psi_{s}+\phi_{s}\right]^{2},
\end{aligned}
$$

where $\omega_{r} \in[0,1]$ is an indicator variable that weights the individual contribution of the data; $\lambda$ and $\mu$ are positive parameters that control the solution smoothness and the outliers detection, respectively. We used $\lambda=0.2$ and $\mu=0.01$ in our experiments. The regularization term involves cliques of size $3,\langle q, r, s\rangle$, that correspond to horizontal, vertical and diagonals pixel triads (see figure 1). Such term is, the well known, thin plate model that penalizes changes in the second derivative of the recovered phase, $\psi+\phi$. 
Given an initial phase $\psi$, and the residual phase $\phi_{r}=0$, for all $r \in R$, The refined phase $\psi+\phi$ is computed with a two step method. In the first step, the weight, $\omega$, and the residual phase, $\phi$, are computed by an alternated minimization of (4); i.e., $U_{1}$ is minimized with respect to (w.r.t.) $\omega$ by keeping $\phi$ fixed and then it is minimized w.r.t. $\phi$ with $\omega$ fixed. The minimization w.r.t. $\omega$ results in the closed formula:

$$
\omega_{r}=\mu /\left[\mu+E^{2}\left(\phi_{r} ; \psi_{r}\right)\right] .
$$

Note that $\omega_{r} \approx 1$ for those sites where the square error $E^{2}\left(\phi_{r} ; \psi_{r}\right)$ is small with respect to $\mu$. On the other hand, $\omega_{r} \approx 0$ for those pixels where the model does not fit very well the data, so that the regularization term has more control over the computation of $\phi$. In the second step, the phase $\psi$ is updated by the computed residual $\phi$, i.e.: $\psi_{r}=\psi_{r}+\phi_{r}$. These two steps are iterated until convergence, such convergence is guaranteed if the computed residual phase, $\phi$, is small, so that:

$$
\|\hat{g}-\hat{b} \cdot * \cos \psi\| \geq\|\hat{g}-\hat{b} \cdot *(\cos \psi-\phi \sin \psi)\| \approx\|\hat{g}-\hat{b} \cdot * \cos (\psi+\phi)\| \geq 0
$$

is satisfied in each iteration, where $*$ denotes the component-wise product of vectors. Note that if a residual phase, $\phi$, increases significantly the error at some pixels, then outliers detection will promotes a smooth reconstruction of the phase at those problematic sites. The details of the phase refinement procedure are formalized in algorithm 1.

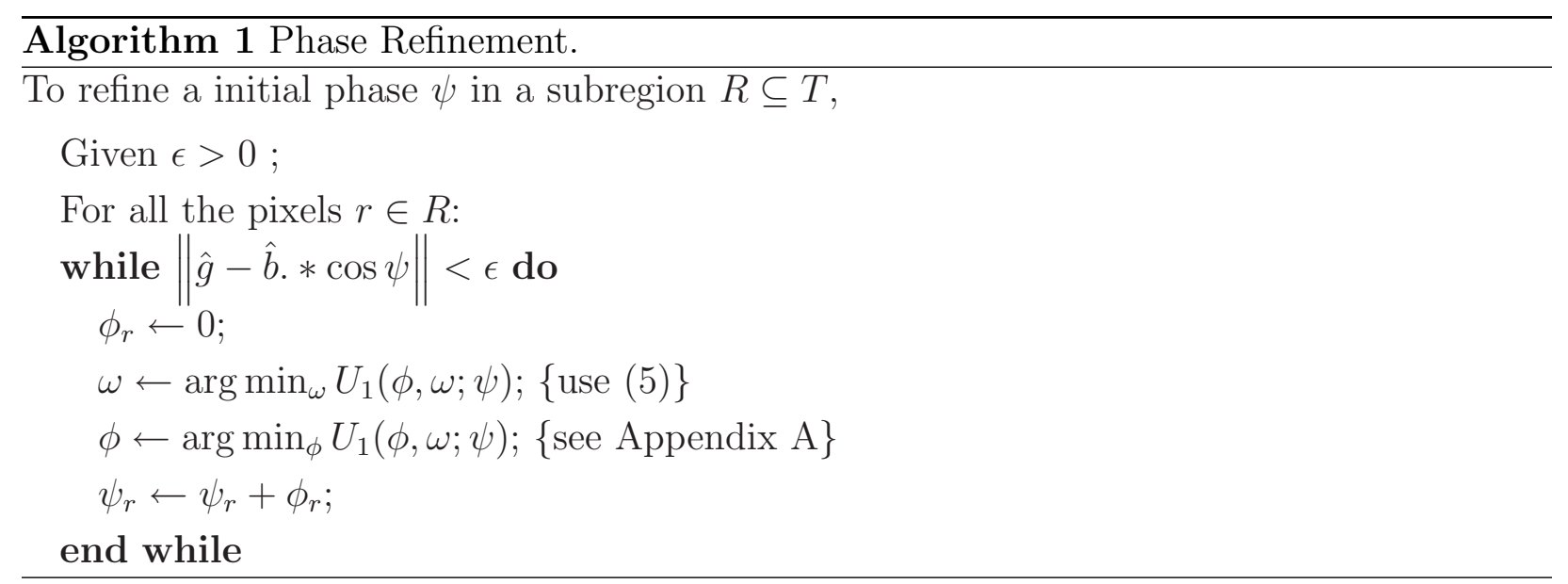

\section{B. Refinement phase experiments}

The performance of the phase refinement method is demonstrated by the experiment illustrated by figure 2. Panel 2-(a) shows an original fringe pattern of a progressive lens generated with a Moire deflectometry setup. Panel 2-(b) shows the wrapped phase computed with the Fourier method. ${ }^{1}$ The region of interest and the wrapped refined phase are shown in panels Panel 2-(d) and 2-(c), respectively. Figure 3 shows a detail of the results in figure 2. Note 
that the original wrapped phase, panel 3-(a), is distorted by a border effect; such defect is corrected in the refined phase, panel 3-(c). It is important to remark that we show wrapped phases only for visualization purposes: the recovered refined phase is unwrapped.

\section{Closed-Fringe Analysis}

\section{A. Method for closed-fringe analysis}

Now, we extend the refinement phase method for analyzing closed-fringe interferograms. Initially, the seed phase, $\psi$, is available for a small compact region, $R$, of the interferogram (note that $R \subset T$ ). Then we define the region $S$ that contains the pixels located in a narrow band (with width defined by $d$ ) around $R$ :

$$
S=\{s \in T \mid s \notin R, r \in R,\|r-s\|<d\} .
$$

We used $d=2$ in all the experiments. The initial phase $\psi$ in $R$ can be computed with a method for opened-fringe patterns in a small region with such characteristics. Once the initial conditions are established $(\psi, \phi=0, R$ and $S)$, we compute and propagate the phase using again an iterative strategy of two steps. In the first step, we refine of the phase $\psi+\phi$ in $R$. Then, we grow $R$ in, at least, one pixel in the second step. The phase refinement is achieved using the previously presented method [based on (4)]. As it is known, RPT method ${ }^{9,14,12}$ may produce a wrong phase due to an unsuccessful normalization of the fringe pattern. In our approach, the grown pixels are chosen such that they minimize the risk of growing a wrong phase. Our growing phase strategy is detailed below.

Once the step of phase refinement is performed, we proceed to grow the region $R$. First, $\psi$ is extrapolated to those pixels in $S$ by minimizing a cost function,

$$
U_{2}(\psi)=\sum_{\langle q, r, s\rangle:\{q, r, s\} \cap S \neq \emptyset}\left(\psi_{q}-2 \psi_{r}+\psi_{s}\right)^{2}
$$

that promotes an extrapolation with constant slope and keeps fixed the values of $\psi$ in $R$. Afterward, the region $R$ is grown by moving some pixels from $S$ (at least one). The selection of a candidate pixel, $r \in S$ to be included in $R$, is done taking into account: a) the confidence of the extrapolated phase, b) the number of its neighbor pixels in $R$ and their confidence and finally c) $R$ is grown preferably along the fringes (i.e. the extrapolated phase is almost constant). In order to implement such constraints, we compute a "confidence measure", $\hat{\omega} \in[0,1]$, by minimizing the cost function:

$$
U_{3}(\hat{\omega})=\sum_{r \in S}\left\{\left[\hat{\omega}_{r}^{2} E^{2}\left(\psi_{r}\right)+\mu\left(1-\hat{\omega}_{r}\right)^{2}\right]+\hat{\lambda} \sum_{s \in N_{r}} b_{r s}\left(\hat{\omega}_{r}-\hat{\omega}_{s}\right)^{2}\right\}
$$


w.r.t. $\hat{\omega}$ by keeping fixed $\psi$ and $\phi$; where $N_{r}=\{s|s \in R \cup S| r-s \mid,<2\}$ is the set of first eight neighbor pixels to $r, b_{r s} \in[0,1]$ is a weight factor that measures the alignment of the pixel pair $\langle r, s\rangle$ with the local fringe and $\hat{\lambda}$ is a positive regularization parameter. The third term in (8) produces an anisotropic smoothing of $\hat{\omega}$ along the fringes. The weights, $b_{r s}$, are directly computed from the fringe pattern with:

$$
b_{r s}=\exp \left[-(r-s)^{T} J_{r}(r-s)\right]
$$

where

$$
J=\operatorname{trace}\left(\tau \tau^{T}\right) I-\tau \tau^{T},
$$

is the local inertia tensor; ${ }^{21}$ with $\tau \stackrel{\text { def }}{=}\left[\hat{g}_{x \sigma}, \hat{g}_{y \sigma}\right]^{T}$, where $\hat{g}_{l \sigma}$ denotes the $l$-directional derivative smoothed with a Gaussian kernel (defined by $\sigma$ ) and $I$ is the identity matrix. Note that for $\hat{\lambda}=0$, the minimizer (8) corresponds to (5). Next, we effectively grow the region, $R$, where the phase can be computed by including the immediate neighbor pixels with high confidence. The smoothing of $\hat{\omega}$ and a constrain on the support of grown pixels promote smooth wavefronts and avoid the propagation to be trapped in local minimum. As saddle and stationary points may induce the algorithm to produce a wrong phase, we implement a scanning strategy that leaves such problematic sites for the last, once the surrounding pixels have been demodulated. This scanning strategy is based on the assumption that the fringe patterns are locally monochromatic: just one frequency is present in a small region. That is, the fringe patterns have a well defined local structure: small directional derivatives along the fringes and large ones across them, except in problematic sites. This growing scheme is implemented with the updating formula:

$$
R^{n+1}=R^{n} \cup\left\{r \in S \mid c_{r} \cdot \hat{\omega}_{r}>\theta, \operatorname{card}\left(N_{r} \cap R^{n}\right) \geq 2\right\},
$$

where

$$
c_{r}=\left(\lambda_{1 r}-\lambda_{2 r}\right) /\left(\lambda_{1 r}+\lambda_{2 r}\right)
$$

is the local coherency that can be understood as a normalization of $\left\|\nabla f_{r}\right\| ; \lambda_{1} \geq \lambda_{2}$ are the eigenvalues of the inertia tensor, $J$, defined in (10) and the cardinality operator, $\operatorname{card}(\cdot)$, returns the number of elements in a set. Then, a pixel is grown if at least 2 neighboring pixels are in $R$ and its weighted confidence, $c_{r} \cdot \hat{\omega}_{r}$, pass a threshold, $\theta$; if no pixel pass the threshold, then the one with largest $c_{r} \cdot \hat{\omega}_{r}$ is selected. The growing of $R$ completes an iteration of wavefront propagation. The iterations continue until the phase is estimated in the region of interest: $R=T$.

It is important to note that, in the closed fringe analysis method, the phase refinement step can be performed in a narrow band close to the border between $R$ and $S$ (we use a band 3 pixels wide) with a subsequent reduction of the computational time. The details of the closed fringe method are formalized in algorithm 2. 


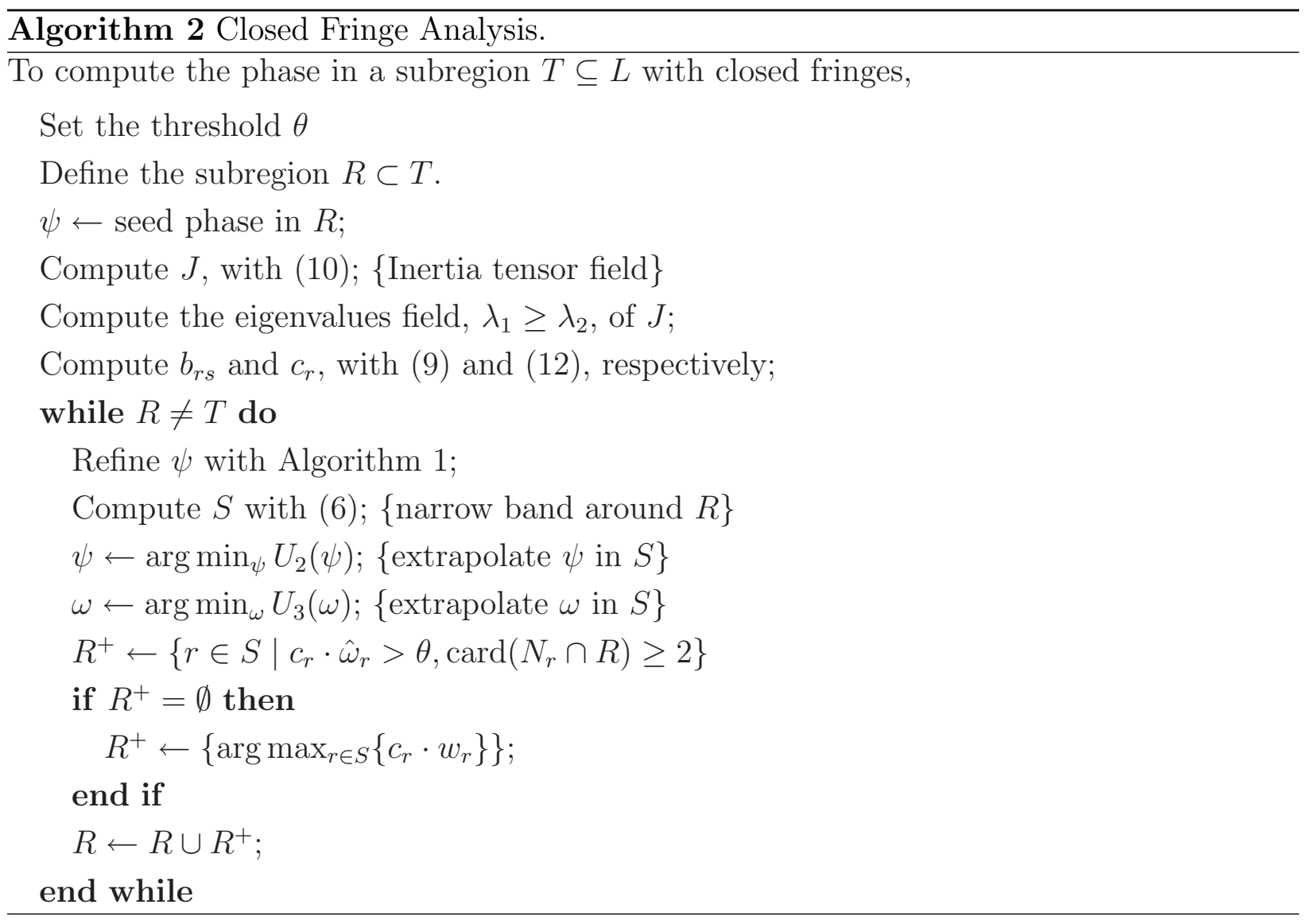




\section{B. Closed-fringe analysis experiments}

In order to illustrate the algorithm behavior, we show a sequence of the phase propagation in figure 4. The synthetic fringe pattern is shown in top-left panel and the final recovered phase is shown in bottom-right panel. One can observe that: sites without a well-defined orientation (as the fringe centers) are leaved for the least, the phase propagation is preferably performed along the fringes and such propagation has a smooth wavefront. These characteristics reduce the risk of computing a wrong phase.

Figure 5 shows the result of an experiment designed to demonstrates the performance of the closed fringe analysis method. Panel ??-(a) shows the normalized fringe pattern of a real ESPI image. The fringe pattern shows the relative deformation of a steel plate when a thermal stress is applied. For illustration purposes, we show in panel 5-(b) plots of the gray level of the fringes. These plots corresponds to the row (top) and the column (bottom) that crossing at the minimum phase (fringe center). Panel $5-(\mathrm{c})$ shows the binarization of the fringes. As one can see, the normalization process is not successfully achieved as is appreciated in the figure and the fringes are not correctly distinguished. Therefore, a scanning strategy that follow fringes will compute a wrong phase. However, our method estimates the right phase in spite of the fact that we set $\hat{b}=1$ in the whole domain. Panel 5-(d) shows the computed coherency map that defines the local threshold in (11). Panel 5-(e) shows the computed phase with the proposed method and the rewrapped phase is shown in panel 5-(f).

For comparison purposes, figure 6 presents the rewrapped phase of results computed with: the RPT method reported in ${ }^{9}$ [panel 6-(a)] and the RPT variant with the joint estimation of the phase, $f$, and the contrast, $b .{ }^{12}$ The dynamic range of the unwrapped phases are about 3.0 and 15.0 radians for the phases in panels 6 -(a) and 6-(b), respectively. In spite of the improvement in the computed phase with the Legarda et al. method, the contrast term, $b$, with frequency bandwidth closed to the fringe pattern mislead the joint estimation of phase and contrast.

\section{Conclusions}

First, we presented a phase refinement method that improves phase computed, initially, from a fringe pattern with incomplete domains. The method is based on the iterative minimization of a robust half-quadratic cost function with explicit outliers rejection. Such minimization is achieved by alternately solving a liner system and computing a closed formula.

In second part of the paper, we generalized the phase refinement method in order estimate the phase from closed fringe patterns. This generalization is based on a successive growth of the region of phase refinement. The method has shown, in real experiments, to be robust for analyzing fringes with spatial variable illumination conditions (see experiment of figure 5) .

It is important to remark that both algorithms are based in the minimization of quadratic 
cost functions (with linear residuals) that allows us to use simple, fast and stable minimization algorithms. The outliers detection improves the performance of the methods for recovering phases from noisy fringe patterns or in the case that the initial seed phase had regions with large residuals.

\section{Acknowledges}

The author thanks to J.L. Marroquin and R. Legarda-Saenz for their comments that improved the quality of the paper. This work was partially supported by CONACYT (Mexico) under grant 40722Y1. The author's email is mrivera@cimat.mx.

\section{Appendix A. Gauss-Seidel equations for computing the refined phase}

The computation of refined phase $\phi$ in Algorithm 1 is computed by minimizing (4) w.r.t. $\phi$, keeping fixed $\omega$. Such quadratic minimization can be achieved with many algorithms. In this appendix we present the detailed equation of a Gauss-Seidel scheme. The componentwise update formula is:

$$
\phi_{i j}=\frac{\omega_{i j}^{2} \sin \psi_{i j}\left[\hat{b}_{i j} \cos \psi_{i j}-g_{i j}\right]+\lambda V(i, j)}{\omega_{i j}^{2} \hat{b}_{i j} \sin ^{2} \psi_{i j}+\lambda W(i, j)},
$$

with the follow definitions:

$$
\begin{gathered}
V(i, j) \stackrel{\text { def }}{=} \sum_{c=-1}^{1}\left[P_{1}(i-c, j)+P_{2}(i, j-c)+P_{3}(i-c, j-c)+P_{4}(i-c, j+c)\right], \\
W(i, j) \stackrel{\text { def }}{=} \sum_{c=-1}^{1}\left[N_{1}^{2}(i-c, j)+N_{2}^{2}(i, j-c)+N_{3}^{2}(i-c, j-c)+N_{4}^{2}(i-c, j+c)\right] ;
\end{gathered}
$$

where

$$
\begin{aligned}
& P_{m}(k, l) \stackrel{\text { def }}{=} \phi_{i j} N_{m}^{2}(k, l)-Q_{m}(k, l) N_{m}(k, l), \\
& N_{m}(k, l) \stackrel{\text { def }}{=} A_{m}(k, l) \frac{\partial}{\partial \phi_{i j}} Q_{m}(\phi, k, l) ;
\end{aligned}
$$


and

$$
\begin{aligned}
& A_{1}(k, l)=\left\{\begin{array}{l}
1 \text { if }\langle(k-1, l),(k, l),(k+1, l)\rangle \in R \\
0 \quad \text { otherwise, }
\end{array}\right. \\
& A_{2}(k, l)=\left\{\begin{array}{l}
1 \text { if }\langle(k, l-1),(k, l),(k, l+1)\rangle \in R \\
0 \quad \text { otherwise, }
\end{array}\right. \\
& A_{3}(k, l)=\left\{\begin{array}{l}
1 \text { if }\langle(k-1, l-1),(k, l),(k+1, l+1)\rangle \in R \\
0 \quad \text { otherwise, }
\end{array}\right. \\
& A_{4}(k, l)=\left\{\begin{array}{l}
1 \text { if }\langle(k-1, l+1),(k, l),(k+1, l-1)\rangle \in R \\
0 \quad \text { otherwise, }
\end{array}\right. \\
& Q_{1}(k, l) \stackrel{\text { def }}{=} \psi_{k-1, l}+\phi_{k-1, l}-2\left(\psi_{k, l}+\phi_{k, l}\right)+\psi_{k+1, l}+\phi_{k+1, l}, \\
& Q_{2}(k, l) \stackrel{\text { def }}{=} \psi_{k, l-1}+\phi_{k, l-1}-2\left(\psi_{k, l}+\phi_{k, l}\right)+\psi_{k, l+1}+\phi_{k, l+1} \text {, } \\
& Q_{3}(k, l) \stackrel{\text { def }}{=} \psi_{k-1, l-1}+\phi_{k-1, l-1}-2\left(\psi_{k, l}+\phi_{k, l}\right)+\psi_{k+1, l+1}+\phi_{k+1, l+1} \text {, } \\
& Q_{3}(k, l) \stackrel{\text { def }}{=} \psi_{k-1, l+1}+\phi_{k-1, l+1}-2\left(\psi_{k, l}+\phi_{k, l}\right)+\psi_{k+1, l-1}+\phi_{k+1, l-1} \text {. }
\end{aligned}
$$




\section{References}

1. M. Takeda, H. Ina and S. Kobayashi, "Fourier-transform method of fringe-pattern analysis for computer-based topography and interferometry," J. Opt. Soc. Am. A, 4, 156-160 (1982).

2. K. H. Womack, "Interferometric phase measurement using spatial synchronous detection," Opt. Eng. 23, 391-395 (1984).

3. D.W. Robinson and G. T. Reid, eds. Interferogram Analysis: Digital Fringe pattern Measurement Techniques (Institute of Phisics, Bristol, 1993).

4. J.L. Marroquin, J.E. Figueroa and M. Servin, "Robust quadrature filters," J. Opt. Soc. Am. A, 14, 4, 779-791 (1997).

5. J.L. Marroquin, M. Servin and R. Rodriguez-Vera, "Adaptive quadrature filters and the recovery of phase from fringe pattern images," J. Opt. Soc. Am. A, 14, 8, 1742-1753 (1997).

6. M. Servin, J.L. Marroquin, and F. J. Cuevas, "Demodulation of a single interferogram by use of a two-dimensional regularized phase-tracking technique," Appl. Opt. 36, 45404548 (1997).

7. J. L. Marroquin, R. Rodriguez-Vera and M. Servin, "Local phase from local orientation by solution of a sequence of linear systems," J. Opt. Soc. Am. A, 15, 6, 1536-1544 (1998)

8. J. Villa, J.A. Quiroga, and M. Servin, "Improved regularized phase tracking technique for the processing of squared-grating deflectograms" Appl. Opt. 39, 502-508 (2000).

9. M. Servin, J.L. Marroquin and F.J. Cuevas, "Fringe-follower regularized phase tracker for demodulation of closed-fringe interferograms," J. Opt. Soc. Am. A, 18, 3, 689-695 (2001).

10. K.G. Larkin, D. Bone, and M. A. Oldfield, "Natural demodulation of two-dimensional fringe patterns. I. General background of the spiral phase quadrature transform," J. Opt. Soc. Am. A 18, 1862-1870 (2001).

11. K.G. Larkin, "Natural demodulation of two-dimensional fringe patterns. II. Stationary phase analysis of the spiral phase quadrature transform," J. Opt. Soc. Am. A 18, 8, 1871-1881 (2001).

12. R. Legarda-Saenz, W. Osten and W. Jüptner, "Improvement of the Regularized Phase Tracking Technique for the Processing of Nonnormalized Fringe Patterns ", Appl, Opt. 41, 5519-5526 (2002).

13. M. Servin, J. A. Quiroga and J. L. Marroquin, "General n-dimensional quadrature transform and its application to interferogram demodulation," J. Opt. Soc. Am. A 20, 5, 925 (2003)

14. M. Servin, J.L. Marroquin and J.A. Quiroga, "Regularized quadrature and phase track- 
ing from a single closed-fringe interferogram," J. Opt. Soc. Am. A, 21, 411-419 (2004).

15. D. Geman and G. Reynolds, "Constrained restoration and the recovery of discontinuities," IEEE Trans. Image Process., 14, 367-383 (1992).

16. M.J. Black and A. Rangarajan, "Unification of line process, outlier rejection, and robust statistics with application in early vision," Int. J. Comput. Vis., 19, 57-91 (1996).

17. P. Charbonnier, L. Blanc-Féraud, G. Aubert and M. Barlaud, "Deterministic edgepreserving regularization in computer imaging," IEEE Trans. Image Process, 6, 298-311 (1997).

18. M. Rivera and J.L. Marroquin, "Adaptive rest condition potentials: Second order edgepreserving regularization," Comput. Vision Image Understand., 88, 76-93 (2002).

19. M. Rivera, and J.L. Marroquin, "Efficient half-quadratic regularization with granularity control," Image and Vision Computing, 21, 345-357 (2003).

20. M. Rivera and J.L. Marroquin, "Half-quadratic cost functions for phase unwrapping," Opt. Lett. 29, 504-506 (2004).

21. B. Jahne, Digital Image Processing (Springer Verlag Berlin, 5th ed., 2002). 


\section{List of Figure Captions}

Figure 1. Cliques with triads of pixels $\langle q, r, s\rangle$.

Figure 2. Phase refinement. (a) Fringe pattern. (b) Approximated phase. (c) Mask. (d) Computed refined phase. The phases in (b) and (d) are rewrapped for display purposes.

Figure 3. Details of the phase refinement, the illustrated region corresponds to a rectangular region $(80 \times 80$ pixels $)$ located at the left and button of the panels $2-b, 2-c$ and $2-d$, respectively. (a) Approximated phase. (b) Mask. (d) Refined phase.

Figure 4. Phase propagation sequence. Top-left panel: synthetic fringe, sequence: phase propagation.

Figure 5. Closed fringe analysis. (a) Fringe pattern. (b) Gray scale plot of the row (top) and the column (bottom) that crossing at the fringe center. (c) Binary map of fringes. (d) Coherency map. (e) Computed phase. (f) Rewrapped phase, for display purposes.

Figure 6. Phase tracker reconstructions with (a) RPT reported in ref. ${ }^{9}$ (b) RPT with join estimation of the phase, $f$, and the contrast, $b .^{12}$ 


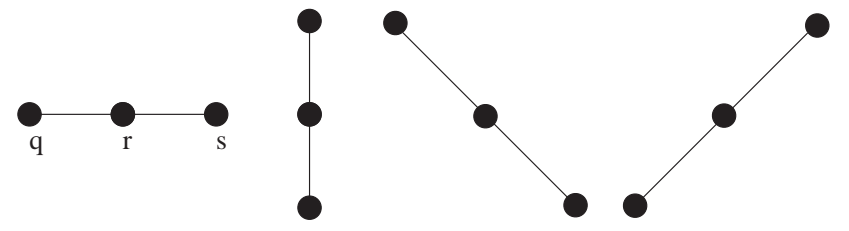

Fig. 1. Cliques with triads of pixels $\langle q, r, s\rangle$. riveraf1.eps. 


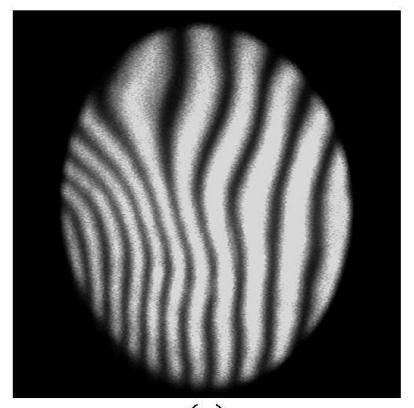

(a)

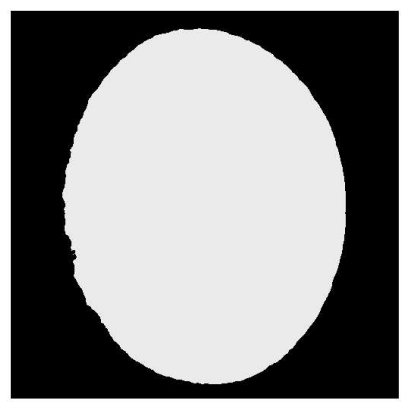

(c)

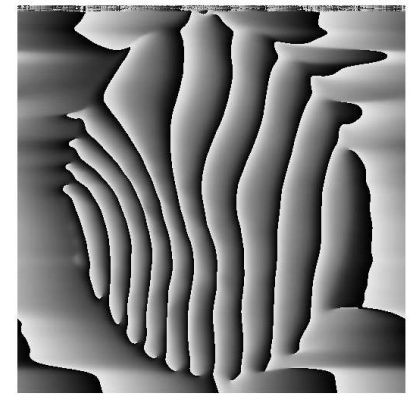

(b)

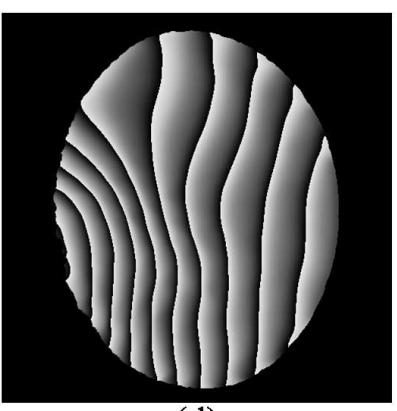

(d)

Fig. 2. Phase refinement. (a) Fringe pattern. (b) Approximated phase. (c) Mask. (d) Computed refined phase. The phases in (b) and (d) are rewrapped for display purposes. riveraf2.eps. 


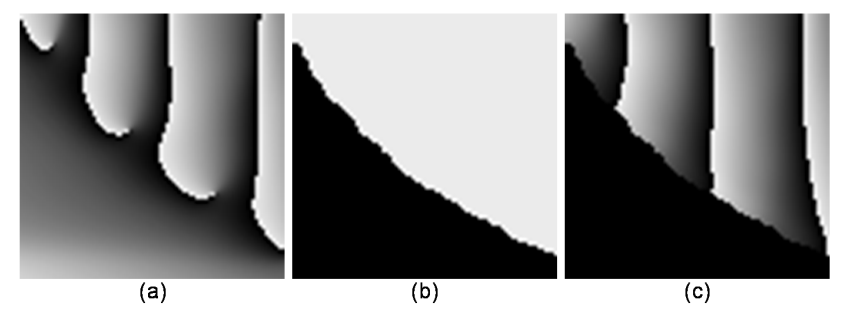

Fig. 3. Details of the phase refinement, the illustrated region corresponds to a rectangular region $(80 \times 80$ pixels $)$ located at the left and button of the panels 2-b, 2-c and 2-d, respectively. (a) Approximated phase. (b) Mask. (d) Refined phase. riveraf3.eps. 


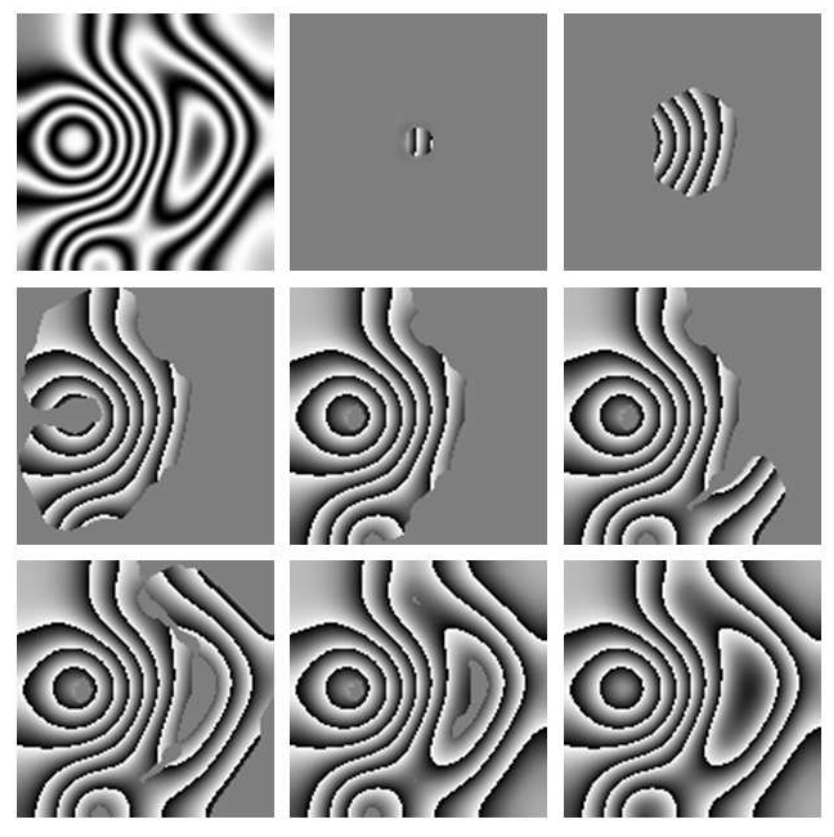

Fig. 4. Synthetic fringe pattern (top-left) and a computed phase propagation sequence. riveraf4.eps. 


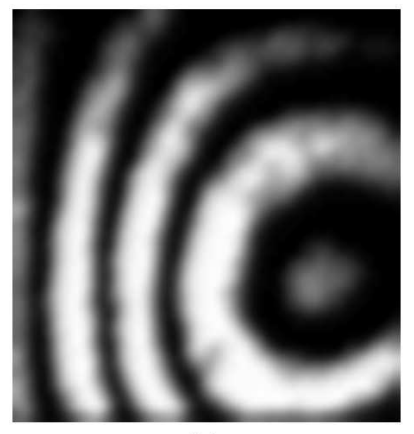

(a)

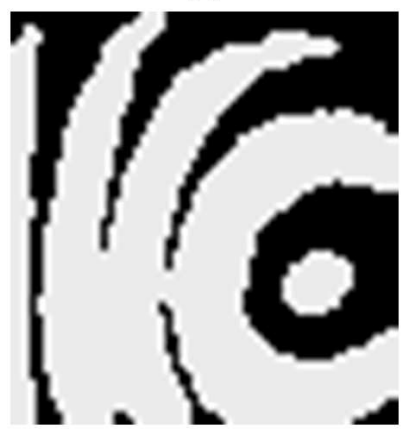

(c)

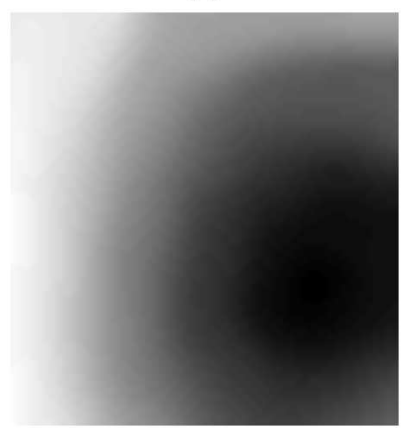

(e)

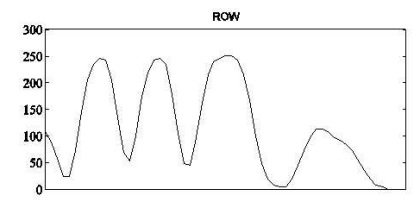

COLW

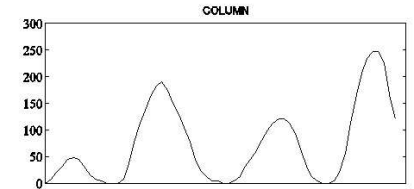

(b)

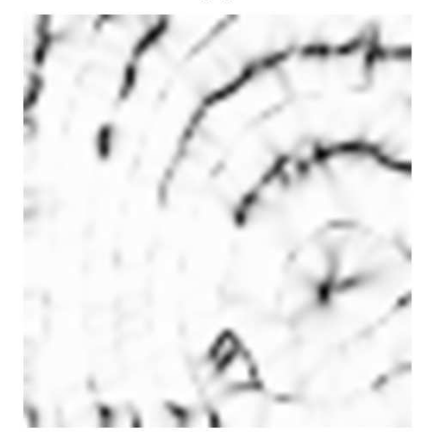

(d)

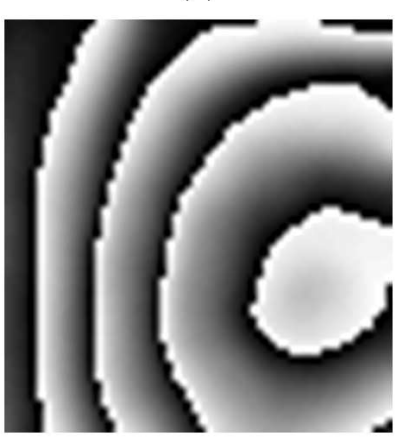

(f)

Fig. 5. Closed fringe analysis. (a) Fringe pattern. (b) Gray scale plot of the row (top) and the column (bottom) that crossing at the fringe center. (c) Binary map of fringes. (d) Coherency map. (e) Computed phase. (f) Rewrapped phase, for display purposes. riveraf5.eps. 


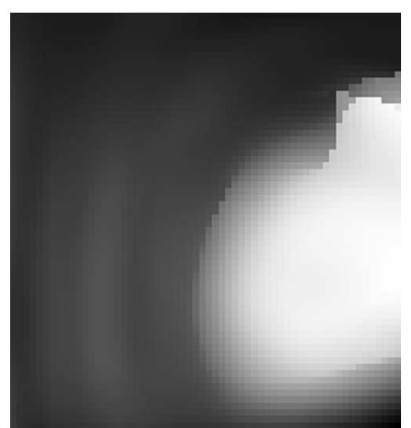

(a)

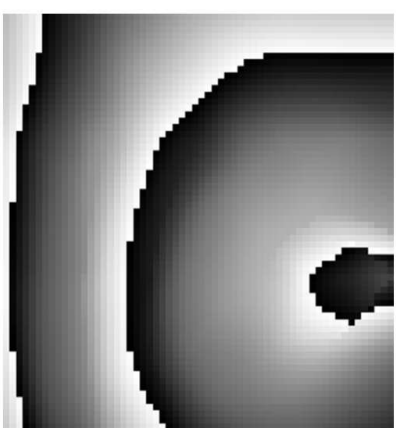

(b)

Fig. 6. Phase tracker reconstructions with (a) RPT reported in ref. ${ }^{9}$ (b) RPT with join estimation of the phase, $f$, and the contrast, $b{ }^{12}$ riveraf6.eps. 